\title{
Combining Ability Studies for Morpho-Physiological, Yield, Yield Attributes Nitrogen Use Efficiency and Its Related Traits in Bread Wheat (Triticum aestivum L.)
}

\author{
Mahalaxmi K. Patil and S.A. Desai* \\ Department of Genetics and Plant Breeding, College of Agriculture, University of \\ Agricultural Sciences, Dharwad 580 005, India \\ *Corresponding author
}

\begin{tabular}{|c|c|}
\hline & \multirow{6}{*}{$\begin{array}{l}\text { In India, wheat is the most important cereal crop and plays a major role in food and nutritional } \\
\text { safety. Worldwide, there is a growing interest in the development of wheat cultivars that are more } \\
\text { effective in utilizing nitrogen (N) and better suited to N constraints. he objective of this research was } \\
\text { to investigate the comparative significance of general (GCA) and specific (SCA) combining ability } \\
\text { in a set of wheat genotypes with promising lines and their corresponding half-diallel crosses. } \\
\text { Combining yield ability for yield attributes, nitrogen use efficiency (NUE) and its associated } \\
\text { characters were studied in twelve bread wheat genotypes and their sixty six } \text { F }_{1} \text { s derived from half- } \\
\text { diallel crosses and assessed under } 50 \text { per cent of recommended dose of nitrogen condition using } \\
\text { three replicated alpha lattice design. Variance due to GCA has been found to be important for all } \\
\text { traits. This indicates the significance of additive genetic variance in regulating the expression of } \\
\text { these characters. Significance of variance due to SCA was noted for all traits except for grain protein } \\
\text { content and nitrogen harvest index, showing the significance of non-additive genetic variance in } \\
\text { regulating the expression of these characters. In most of the traits, the variance ratios were less than } \\
\text { unity except for the grain protein content and nitrogen harvest index, thus representing the } \\
\text { preponderance of non-additive gene action for most characteristics. Parents namely, } 3 \text { SATYN } 9402 \text {, } \\
\text { K9107, HD } 2967 \text { and } 46 \text { IBWSN } 1005 \text { were the best general combiners and crosses namely, } 3 \\
\text { SATYN } 9402 \times \text { K9107, 3 SATYN } 9402 x \text { HD } 2967,3 \text { SATYN } 9402 x 46 \text { IBWSN } 1005,3 \text { SATYN } \\
9402 \times \text { DBW } 14 \text {, HD } 2967 x 46 \text { IBWSN } 1005 \text { and WH } 1022 x \text { K9107 found to be the best specific } \\
\text { combiners for yield and NUE associated traits. }\end{array}$} \\
\hline Keywords & \\
\hline & \\
\hline Article Info & \\
\hline & \\
\hline & \\
\hline
\end{tabular}

A B S T R A C T

\section{Introduction}

Wheat (Triticum spp.) is the major cereal grain in the world and belongs to the Graminae family. Due to the acreage it occupies, high productivity and the prominent position it maintains in global food grain trade, it has been defined as the' King of cereals.' India is the world's second-largest wheat producer after China and the country's second-largest food crop. Wheat played a crucial role in stabilizing the country's food grain production. As land and water resources are limited, the rise in wheat production can only be accomplished by raising the average yield. Moreover, enhanced agricultural production around the globe as a result of the green revolution was primarily based on the use of mineral fertilizers, which resulted in an rise in worldwide $\mathrm{N}$ fertilizer consumption from 10.8 
million tonnes in the year 1960 to 85.6 million tonnes in the year 2000, and is expected to raise to 240 million tonnes by 2050 (Crews and Peoples, 2004).

The efficiency with which plant uses $\mathrm{N}$, a lot of attention has been paid to the nitrogen use efficiency (NUE). NUE may be described as the proportion of grain yield or biomass by the nitrogen supply available in the soil (Moll et al., 1982). According to Moll et al., (1982), $\mathrm{N}$ use efficiency (NUE) in cultivar growth can be split into two components: (i) uptake efficiency (UPE) (complete plant $\mathrm{N} / \mathrm{N}$ supplied), which is the ability of the crop to extract $\mathrm{N}$ from the soil; and (ii) use effectiveness (UTE) (grain yield / total plant $\mathrm{N}$ ), which measures the plant's ability to transform the already absorbed $\mathrm{N}$ into grain yield in the plant. Plants ability to capture $\mathrm{N}$ from the soil relies on factors including type of soil, climate, and species. Genetic variability for both $\mathrm{N}$ uptake efficiency and $\mathrm{N}$ utilization efficiency exists for a large amount of crop plants (Hirel et al., 2007). In addition, the occurrence of interactions between the genotype and $\mathrm{N}$ level resulted to the conclusion that the best performing crop varieties at high $\mathrm{N}$ fertilization input are not necessarily the best varieties at reduced $\mathrm{N}$ supply (Gallais and Coque, 2005).

Agricultural crops are inefficient in the uptake and utilization of fertilizer nitrogen, including wheat. Reports have shown that the nitrogen use efficiency NUE) in wheat is around 40-50 percent, resulting in the loss of more than 50 per cent of the applied fertilizer nitrogen to the environment and a potential source of pollution and extra costs for farmers (Barraclough et al., 2010, Gorny et al., 2011 and Hawkesford, 2012). Increasing emphasis on breeding wheat cultivars with enhanced NUE to reduce excessive nitrogen fertilizer input and maintain an acceptable yield is therefore a worldwide issue (Hirel et al., 2007 and Foulkes et al., 2009). As a result, NUE enhancement will simultaneously boost farmers ' earnings and decrease the adverse environmental impact of $\mathrm{N}$ loss on the environment and its food production impact. Nitrogen-use efficient crop varieties may be a great choice for ensuring sustainability in agricultural systems and meeting future customer requirements, particularly in the face of changing climate change environments.

It is of great importance to improve the nitrogen use efficiency and yield potential of the wheat varieties information on the genetic mechanisms of the wheat varieties, such combining ability. Often breeders face issues in selecting those parental lines, which can provide greater frequency of most desirable segregants in different cross combinations. To achieve this goal, knowledge of prepotency of parental lines for combining ability is very useful in selecting desirable lines (Ashadusjam et al., 2012). In view of the above facts, the current experiment has been undertaken to estimate the combining ability effects of different parents and crosses in F1 population and to determine the additive and non-additive genetic effects of yield and NUE related traits.

\section{Materials and methods}

Twelve diverse parents, 2 WYCYT 34, WH 1022, 5 CISA HTEM 10212, GW 322, 3 SATYN 9402, K9107, HD 2967, RAJ 1972, PBW 343, 5 CISA HTEM 10211, 46 IBWSN 100 , DBW 14 were chosen as parents on the grounds of their yield potential and NUE component traits such as harvest index, nitrogen uptake efficiency, nitrogen utilization efficiency and NUE etc. These parents were crossed in all possible combinations excluding reciprocals to generate sixty-six $\mathrm{F}_{1}$ hybrids consisting of experimental components for this experiment and the experiment was carried out with three replications in alpha 
lattice design. At All India Coordinated Wheat Improvement Project, University of Agricultural Sciences, Dharwad, all the $\mathrm{F}_{1}$ hybrids along with their parents were grown in 6 rows of 3 meter long bed with a spacing of $20 \mathrm{~cm}$ between rows and plant to plant distance within row was $10 \mathrm{~cm}$ under 50 per cent of the recommended dose of nitrogen $(\mathrm{N})$ during rabi 2017-18.

Twenty one traits namely, chlorophyll content at booting stage, anthesis stage and grain filling stage, days 50 per cent heading, days to maturity, plant height, number of productive tillers per plant, spike length, awn length, number of spikelets per spike, number of grains per spike, thousand grain weight, grain yield, biomass, harvest index, grain protein content (\%), nitrogen harvest index, total nitrogen uptake, nitrogen uptake efficiency, nitrogen utilization efficiency and nitrogen use efficiency were recorded on randomly chosen competitive parent plants and from each replication in parents and F1s. The data was analyzed for combining capacity according to Griffing's Method 2, Model I (1956).

\section{Results and Discussion}

\section{Analysis of variance for combining ability}

The analysis of variance for combining ability for morpho-physiological, yields, yield attributes, NUE and its associated traits is presented in (Table 1). ANOVA for combining ability revealed that variance due to general combining ability (GCA) was found to be significant for all the attributes. This indicated the importance of genetic additive variance in regulating the expression of these characters. Significance of variance owing to specific combination ability (SCA) was noted for all characters except protein and nitrogen harvest index, showing the significance of non-additive genetic variance in regulating the expression of these characters. With the exception of protein and nitrogen harvest index, the variance proportions were less than unity in most of the attributes, thus representing the preponderance of dominant gene action (presence of more non-additive gene action) for most characters.

Likewise, Singh et al., (2012) and Ranjitha et al., (2018) also recorded non-additive variance in expression of quantitative characters. In this research, the preponderance of dominance (non-additive) effects indicated that selection techniques that eliminate non-additive masking effects and take benefit of the additive variance should be used to enhance nitrogen efficiency characteristics in the low$\mathrm{N}$ environment.

\section{General combining ability effects of the parents}

Estimate of gca effects of the parents for different yield, yield attributes, NUE and related traits are presented in Table 2 .

General Combining Ability (GCA) was defined by Sprague and Tatum (1942) as the average performance of line or tester in series of hybrid combinations. Among twelve parents The NUE parents namely, 3 SATYN 9402, K9107, HD 2967 and 46 IBWSN 1005 manifested significant and positive gca effects for most of the attributes (mainly yield, Harvest index, NUtE, NUE) studied.

These parents were not only good general combiners but their per se performance was also superior for most of the characters studied. For future breeding programmes, genotypes with high GCA values for economically valuable characteristics such as grain yield, NUE and its related components should be used to boost the wheat yield. Mahantashivayogayya et al., (2004), Joshi et al., (2004), Khan et al., (2007), Ranjitha et al., (2018) revealed similar outcomes. 
Table.1 ANOVA for combining ability for morpho-physiological, yield, yield attributes, NUE and related traits in $12 \times 12$ half diallel crosses

\begin{tabular}{|c|c|c|c|c|c|c|c|c|c|c|c|c|}
\hline Character & df & $\begin{array}{l}\text { Chlorophyll } \\
\text { content at } \\
\text { booting } \\
\text { stage }\end{array}$ & $\begin{array}{c}\text { Chlorophy } \\
\text { Il content } \\
\text { at anthesis } \\
\text { stage }\end{array}$ & $\begin{array}{c}\text { Chlorophyll } \\
\text { content at } \\
\text { grain filling } \\
\text { stage }\end{array}$ & $\begin{array}{l}\text { Days to } \\
\text { heading }\end{array}$ & $\begin{array}{l}\text { Days to } \\
\text { maturity }\end{array}$ & Plant height & $\begin{array}{c}\text { Number of } \\
\text { productive } \\
\text { tiller per } \\
\text { meter }\end{array}$ & $\begin{array}{l}\text { Spike } \\
\text { length }\end{array}$ & $\begin{array}{c}\text { Awn } \\
\text { length }\end{array}$ & $\begin{array}{c}\begin{array}{c}\text { Number } \\
\text { of } \\
\text { spikelet's } \\
\text { per spike }\end{array}\end{array}$ & $\begin{array}{c}\text { Number of } \\
\text { grains per } \\
\text { spike }\end{array}$ \\
\hline GCA & 11 & $13.04 * *$ & $10.38^{* *}$ & $8.19 * *$ & $11.38 * *$ & $75.52 * *$ & $84.99 * *$ & $10.63 * *$ & $2.02 * *$ & $0.99 * *$ & $2.30 * *$ & $20.59 * *$ \\
\hline SCA & 66 & $7.11 * *$ & $8.21 * *$ & $6.63 * *$ & $8.20 * *$ & $22.76 * *$ & $38.63 * *$ & $3.20 * *$ & $1.98 * *$ & $0.81 * *$ & $2.31 * *$ & $20.41 * *$ \\
\hline Error & 151 & 0.62 & 0.57 & 0.55 & 0.25 & 0.36 & 0.67 & 0.73 & 0.10 & 0.05 & 0.32 & 2.80 \\
\hline
\end{tabular}

\begin{tabular}{|c|c|c|c|c|c|c|c|c|c|c|c|}
\hline Character & df & $\begin{array}{c}\text { Thousand } \\
\text { grain } \\
\text { weight }\end{array}$ & Grain yield & Biomass & $\begin{array}{c}\text { Harvest } \\
\text { index }\end{array}$ & $\begin{array}{c}\text { Grain } \\
\text { protein } \\
\text { content }\end{array}$ & $\begin{array}{c}\text { Nitrogen } \\
\text { harvest index }\end{array}$ & $\begin{array}{c}\text { Total } \\
\text { nitrogen } \\
\text { uptake }\end{array}$ & $\begin{array}{c}\text { Nitrogen } \\
\text { uptake } \\
\text { efficiency }\end{array}$ & $\begin{array}{c}\text { Nitrogen } \\
\text { utilization } \\
\text { efficiency }\end{array}$ & Nitrogen use efficiency \\
\hline GCA & 11 & $43.44 * *$ & $12.38 * *$ & $22.67 * *$ & $94.68 * *$ & $1.12 * *$ & $11.53 * *$ & $0.01 * *$ & $0.008 * *$ & $18.27 * *$ & $20.35 * *$ \\
\hline SCA & 66 & $7.52 * *$ & $1.81^{* *}$ & $8.53 * *$ & $10.85 * *$ & 0.16 & 1.50 & $0.001 * *$ & $0.002 * *$ & $3.75^{*}$ & $2.97 * *$ \\
\hline Error & 151 & 2.73 & 0.35 & 2.26 & 1.53 & 0.20 & 1.11 & 0.0004 & 0.001 & 1.32 & 0.58 \\
\hline
\end{tabular}

Table. 2 Estimates of general combining ability effects of 12 parents used in $12 \times 12$ half diallel crosses study

\begin{tabular}{|c|c|c|c|c|c|c|c|c|c|c|c|}
\hline Parents & $\begin{array}{l}\text { Chlorophyll } \\
\text { content at } \\
\text { booting stage }\end{array}$ & $\begin{array}{l}\text { Chlorophyl } \\
\text { l content at } \\
\text { anthesis } \\
\text { stage }\end{array}$ & $\begin{array}{l}\text { Chlorophyll } \\
\text { content at } \\
\text { grain filling } \\
\text { stage }\end{array}$ & $\begin{array}{l}\text { Days to } \\
\text { heading }\end{array}$ & $\begin{array}{l}\text { Days to } \\
\text { maturity }\end{array}$ & $\begin{array}{l}\text { Plant } \\
\text { height }\end{array}$ & $\begin{array}{l}\text { Number } \\
\text { of } \\
\text { productiv } \\
\text { e tiller } \\
\text { per meter }\end{array}$ & $\begin{array}{l}\text { Spike } \\
\text { length }\end{array}$ & Awn length & $\begin{array}{l}\text { Number of } \\
\text { spikelet's } \\
\text { per spike }\end{array}$ & $\begin{array}{l}\text { Number } \\
\text { of grains } \\
\text { per spike }\end{array}$ \\
\hline 2 WYCYT 34 & $-0.79 * *$ & $-0.87 * *$ & $-1.10 * *$ & $-1.54 * *$ & $-4.11 * *$ & $-0.50 *$ & $2.02 * *$ & -0.29 & $-0.33 * *$ & -0.03 & -0.17 \\
\hline WH 1022 & -0.09 & $-0.56 * *$ & $-0.44 *$ & $1.03 * *$ & $1.79 * *$ & $1.96 * *$ & 0.14 & 0.44 & $-0.44 * *$ & $0.37 *$ & $1.14 * *$ \\
\hline 5 CISA HTEM 10212 & $1.39 * *$ & $0.84 * *$ & $0.96 * *$ & $0.44 * *$ & $0.96 * *$ & $1.10 * *$ & 0.33 & -0.30 & 0.02 & 0.16 & 0.40 \\
\hline GW 322 & $-1.27 * *$ & $-0.77 * *$ & $-0.85 * *$ & -0.14 & $2.46 * *$ & -0.38 & $-1.05 * *$ & -0.60 & $-0.14 *$ & 0.10 & 0.31 \\
\hline 3 SATYN 9402 & $2.16 * *$ & $2.16 * *$ & $1.55 * *$ & $0.65 * *$ & $-0.68 * *$ & $2.43 * *$ & $0.69 * *$ & 0.37 & 0.05 & $0.31 *$ & $0.93 *$ \\
\hline K9107 & 0.23 & -0.25 & -0.18 & $0.84 * *$ & -0.28 & $3.02 * *$ & $-0.52 *$ & 0.44 & 0.11 & $-0.54 * *$ & $-1.59 * *$ \\
\hline HD 2967 & -0.24 & -0.20 & -0.27 & $-0.71 * *$ & $-2.94 * *$ & $3.94 * *$ & $0.45 *$ & 0.39 & $0.34 * *$ & $0.60 * *$ & $1.82 * *$ \\
\hline RAJ 1972 & $-0.45 * *$ & 0.07 & 0.09 & $-0.54 * *$ & $1.01 * *$ & $-3.04 * *$ & $-0.83 * *$ & -0.37 & $-0.14 *$ & $-0.41 * *$ & $-1.22 * *$ \\
\hline PBW 343 & $-0.52 * *$ & $-0.64 * *$ & -0.34 & $0.53 * *$ & $1.34 * *$ & $-3.54 * *$ & $-0.60 * *$ & -0.15 & 0.11 & $-0.77 * *$ & $-2.29 * *$ \\
\hline 5 CISA HTEM 10211 & $-0.56 * *$ & $-0.40 *$ & -0.24 & $0.06 * *$ & $1.63 * *$ & $-1.53 * *$ & $-0.98 * *$ & -0.37 & $-0.25 * *$ & -0.20 & -0.58 \\
\hline 46 IBWSN 1005 & $0.59 * *$ & $0.66 * *$ & $0.84 * *$ & $0.89 * *$ & $2.32 * *$ & $-1.14 * *$ & 0.10 & 0.24 & $0.43 * *$ & 0.16 & 0.49 \\
\hline DBW 14 & $-0.44 * *$ & -0.04 & -0.01 & $-1.52 * *$ & $-3.49 * *$ & $-2.32 * *$ & 0.24 & 0.20 & $0.23 * *$ & 0.25 & 0.77 \\
\hline Gi-Gj 95\% & 0.59 & 0.57 & 0.55 & 0.38 & 0.46 & 0.62 & 0.64 & 0.24 & 0.17 & 0.42 & 1.26 \\
\hline Gi-Gj 99\% & $0.78 * *$ & $0.75 * *$ & $0.73 * *$ & $0.50 * *$ & $0.60 * *$ & $0.81 * *$ & $0.85 * *$ & 0.31 & $0.23 * *$ & $0.56 * *$ & $1.66 * *$ \\
\hline
\end{tabular}


Contd...

\begin{tabular}{|c|c|c|c|c|c|c|c|c|c|c|}
\hline Parents & $\begin{array}{c}\text { Thousand } \\
\text { grain } \\
\text { weight }\end{array}$ & Grain yield & Biomass & $\begin{array}{c}\text { Harvest } \\
\text { index }\end{array}$ & $\begin{array}{l}\text { Grain } \\
\text { protein } \\
\text { content }\end{array}$ & $\begin{array}{c}\text { Nitrogen } \\
\text { harvest } \\
\text { index }\end{array}$ & $\begin{array}{c}\text { Total } \\
\text { nitrogen } \\
\text { uptake }\end{array}$ & $\begin{array}{l}\text { Nitrogen } \\
\text { uptake } \\
\text { efficiency }\end{array}$ & $\begin{array}{l}\text { Nitrogen } \\
\text { utilization } \\
\text { efficiency }\end{array}$ & $\begin{array}{l}\text { Nitrogen } \\
\text { use } \\
\text { efficiency }\end{array}$ \\
\hline 2 WYCYT 34 & $-0.93 *$ & $-0.40 * *$ & $3.15 * *$ & $-3.57 * *$ & $-0.37 * *$ & $-1.01 * *$ & $0.01 *$ & $0.02 *$ & $-1.76 * *$ & $0.52 * *$ \\
\hline WH 1022 & $-1.84 * *$ & -0.16 & 0.54 & $-0.97 * *$ & -0.18 & $1.02 * *$ & $-0.01 *$ & $-0.02 *$ & $0.62 *$ & -0.21 \\
\hline $\begin{array}{l}5 \text { CISA HTEM } \\
10212\end{array}$ & $-1.63 * *$ & $-0.43 * *$ & $-0.82 *$ & -0.41 & -0.22 & $-0.60 *$ & $-0.01 *$ & $-0.02 *$ & 0.07 & $-0.55 * *$ \\
\hline GW 322 & $-0.99 *$ & $-1.00 * *$ & -0.74 & $-2.06 * *$ & -0.13 & 0.12 & $-0.02 * *$ & $-0.03 * *$ & $-0.66 * *$ & $-1.28 * *$ \\
\hline 3 SATYN 9402 & $3.94 * *$ & $2.26 * *$ & $0.91 *$ & $4.93 * *$ & $0.58 * *$ & $1.72 * *$ & $0.04 * *$ & $0.06 * *$ & $1.96 * *$ & $2.90 * *$ \\
\hline K9107 & $2.18 * *$ & $1.20 * *$ & 0.09 & $2.90 * *$ & $0.35 * *$ & $1.00 * *$ & $0.02 * *$ & $0.03 * *$ & $1.28 * *$ & $1.54 * *$ \\
\hline HD 2967 & $1.89 * *$ & $0.46 * *$ & $-1.53 * *$ & $2.81 * *$ & $0.24 *$ & 0.23 & 0.002 & 0.003 & $0.95 * *$ & $0.59 * *$ \\
\hline RAJ 1972 & $-1.26 * *$ & $-0.56 * *$ & $0.92 *$ & $-2.24 * *$ & -0.02 & -0.32 & 0.001 & 0.002 & $-1.36 * *$ & $-0.72 * *$ \\
\hline PBW 343 & 0.05 & $-0.52 * *$ & $-0.97 *$ & -0.61 & 0.02 & -0.25 & $-0.01 *$ & $-0.01 *$ & -0.41 & $-0.67 * *$ \\
\hline $\begin{array}{l}5 \text { CISA HTEM } \\
10211\end{array}$ & -0.46 & $-0.60 * *$ & 0.06 & $-1.52 * *$ & -0.03 & -0.23 & $-0.01 *$ & $-0.01 *$ & $-0.94 * *$ & $-0.77 * *$ \\
\hline 46 IBWSN 1005 & -0.76 & $0.43 * *$ & $-1.05 * *$ & $2.33 * *$ & 0.09 & -0.14 & 0.004 & 0.00 & $0.88 * *$ & $0.55 * *$ \\
\hline DBW 14 & -0.19 & $-0.68 * *$ & -0.57 & $-1.60 * *$ & $-0.35 * *$ & $-1.54 * *$ & $-0.02 * *$ & $-0.02 * *$ & $-0.62 *$ & $-0.87 * *$ \\
\hline Gi-Gj 95\% & 1.24 & 0.44 & 1.12 & 0.93 & 0.34 & 0.79 & 0.02 & 0.02 & 0.86 & 0.57 \\
\hline Gi-Gj 99\% & $1.63 * *$ & $0.59 * *$ & $1.48 * *$ & $1.23 * *$ & $0.45 * *$ & $1.04 * *$ & $0.02 * *$ & $0.03 * *$ & $1.14 * *$ & $0.75 * *$ \\
\hline
\end{tabular}


Table. 3 Estimates of specific combining ability effects for sixty-six single cross hybrids used in the 12 x 12 half diallel study

\begin{tabular}{|c|c|c|c|c|c|c|c|c|c|c|c|}
\hline Cross & $\begin{array}{l}\text { Chlorophyll } \\
\text { content at } \\
\text { booting stage }\end{array}$ & $\begin{array}{l}\text { Chlorophyll } \\
\text { content at } \\
\text { anthesis stage }\end{array}$ & $\begin{array}{l}\text { Chlorophyll } \\
\text { content at grain } \\
\text { filling stage }\end{array}$ & $\begin{array}{l}\text { Days to } \\
\text { heading }\end{array}$ & $\begin{array}{l}\text { Days to } \\
\text { maturity }\end{array}$ & $\begin{array}{l}\text { Plant } \\
\text { height }\end{array}$ & $\begin{array}{l}\text { Number of } \\
\text { productive } \\
\text { tiller per meter }\end{array}$ & $\begin{array}{l}\text { Spike } \\
\text { length }\end{array}$ & $\begin{array}{l}\text { Awn } \\
\text { length }\end{array}$ & $\begin{array}{l}\text { Number of } \\
\text { spikelet's } \\
\text { per spike }\end{array}$ & $\begin{array}{l}\text { Number } \\
\text { of grains } \\
\text { per spike }\end{array}$ \\
\hline 2 WYCYT 34 x WH 1022 & -0.346 & 0.48 & 1.32 & $-1.64 * *$ & $-2.78 * *$ & 0.19 & $-2.04 *$ & $-2.12 * *$ & $-1.64 * *$ & 0.78 & 2.41 \\
\hline 2 WYCYT 34 x 5 CISA HTEM 10212 & $1.88 *$ & $1.95 * *$ & -0.11 & -0.38 & $-2.28 * *$ & $4.76 * *$ & 0.77 & $1.48 * *$ & $0.84 * *$ & $3.33 * *$ & $8.82 * *$ \\
\hline 2 WYCYT 34 x GW 322 & -1.23 & $-1.48 *$ & -0.44 & $1.20 *$ & $2.56 * *$ & $7.54 * *$ & 0.49 & $-1.65 * *$ & 0.02 & -0.61 & -1.76 \\
\hline 2 WYCYT 34 x 3 SATYN 9402 & -0.66 & -0.86 & -0.16 & -0.59 & $5.70 * *$ & $-5.83 * *$ & -0.59 & 0.15 & $-0.93 * *$ & -0.15 & -0.38 \\
\hline 2 WYCYT 34 x K9107 & $1.50 *$ & 0.06 & 0.53 & $2.89 * *$ & 0.628 & $-6.07 * *$ & -0.37 & 0.31 & -0.26 & $-1.31 *$ & $-3.86 *$ \\
\hline 2 WYCYT 34 x HD 2967 & -1.40 & -0.56 & -0.78 & $4.77 * *$ & $1.63 * *$ & $-7.52 * *$ & 0.65 & $0.70 *$ & -0.32 & $-1.78 * * *$ & $-5.27 * *$ \\
\hline 2 WYCYT 34 x RAJ 1972 & $1.58 *$ & 0.42 & 0.79 & 0.60 & $1.34 *$ & $-1.78 *$ & $1.60 *$ & $-1.55 * *$ & $-0.84 * *$ & -0.10 & -0.23 \\
\hline 2 WYCYT 34 x PBW 343 & $-1.79 *$ & $-2.11 * *$ & $-3.04 * *$ & $-2.14 * *$ & $-3.32 * *$ & $-2.31 * *$ & $2.03 *$ & -0.34 & $-0.46 *$ & -0.41 & -1.16 \\
\hline 2 WYCYT 34 x 5 CISA HTEM 10211 & $2.46 * *$ & $2.14 * *$ & $1.92 * *$ & $-2.66 * *$ & $-5.27 * *$ & $-2.85 * *$ & $2.41 * *$ & 0.48 & $-1.37 * *$ & -0.98 & -2.87 \\
\hline 2 WYCYT 34 x 46 IBWSN 1005 & -0.82 & -0.85 & -0.99 & $2.17 * *$ & $2.37 * *$ & $4.30 * *$ & 0.34 & -0.42 & $-0.78 * *$ & -0.68 & -1.94 \\
\hline 2 WYCYT 34 x DBW 14 & $-2.40 * *$ & $-2.25 * *$ & $-2.77 * *$ & $2.58 * *$ & $1.84 * *$ & $6.40 * *$ & -0.13 & $2.01 * *$ & 0.12 & 0.90 & 2.79 \\
\hline WH 1022 x 5 CISA HTEM 10212 & -1.43 & $-2.45 * *$ & $-3.54 * *$ & $-2.95 * *$ & $1.49 * *$ & -0.27 & -0.02 & -0.21 & $-0.50 *$ & $1.25 *$ & $3.84 *$ \\
\hline WH 1022 x GW 322 & $-1.80 *$ & -0.50 & $-1.84 * *$ & $-1.71 * *$ & $-1.68 * *$ & $2.37 * *$ & 1.03 & -0.38 & $-0.84 * *$ & -0.68 & -2.07 \\
\hline WH 1022 x 3 SATYN 9402 & 1.44 & 0.05 & 1.23 & $-1.50 * *$ & $-3.54 * *$ & 0.66 & -0.71 & $-2.08 * *$ & -0.03 & $-2.23 * *$ & $-6.69 * *$ \\
\hline WH 1022 x K9107 & $2.67 * *$ & $3.40 * *$ & 0.70 & $-1.02 *$ & 0.39 & $-1.66 *$ & $3.44 * *$ & $6.14 * *$ & -0.02 & $-2.05 * *$ & $-6.17 * *$ \\
\hline WH 1022 x HD 2967 & -1.00 & $-1.45 *$ & 0.32 & -0.14 & -0.94 & $4.52 * *$ & -0.13 & $1.30 * *$ & $1.18 * *$ & $1.48 * *$ & $4.43 * *$ \\
\hline WH 1022 x RAJ 1972 & $-1.99 * *$ & $-3.63 * *$ & $-1.88 * *$ & $5.03 * *$ & $3.44 * *$ & -0.77 & 0.15 & -0.28 & $-0.87 * *$ & 0.49 & 1.46 \\
\hline WH 1022 x PBW 343 x & 1.21 & 1.21 & 0.92 & $2.96 * *$ & $5.77 * *$ & $5.47 * *$ & 0.58 & -0.06 & $0.45 *$ & $2.18 * *$ & $6.53 * *$ \\
\hline WH 1022 x 5 CISA HTEM 10211 & $-1.68 *$ & $-1.59 *$ & $-2.95 * *$ & $5.77 * *$ & $6.82 * *$ & $-9.17 * *$ & 0.30 & $-1.35 * *$ & $0.68 * *$ & -0.39 & -1.18 \\
\hline WH 1022 x 46 IBWSN 1005 & $-3.81 * *$ & $-4.29 * *$ & $-2.46 * *$ & -0.73 & $-3.54 * *$ & $-3.17 * *$ & $-3.11 * *$ & $0.99 * *$ & $0.89 * *$ & 0.59 & 1.75 \\
\hline WH 1022 x DBW 14 & $2.39 * *$ & 0.87 & $1.46 *$ & $3.01 * *$ & $3.94 * *$ & $-3.29 * *$ & -0.25 & 0.35 & $0.92 * *$ & -0.84 & -2.53 \\
\hline 5 CISA HTEM 10212 x GW 322 & $1.95 * *$ & 0.63 & 0.57 & 0.55 & $1.49 * *$ & $-16.43 * *$ & -1.16 & $0.90 * *$ & $0.70 * *$ & $-1.8 * *$ & $-5.33 * *$ \\
\hline 5 CISA HTEM 10212 x 3 SATYN 9402 & $1.73 *$ & $2.15 * *$ & $2.64 * *$ & $3.43 * *$ & $6.63 * *$ & $-11.67 * *$ & 1.44 & $-2.98 * *$ & $-1.91 * *$ & $-3.34 * *$ & $-9.95 * *$ \\
\hline 5 CISA HTEM 10212 x K9107 & 0.03 & -0.05 & -0.53 & $4.58 * *$ & $3.56 * *$ & $-8.43 * *$ & -0.02 & -0.14 & $0.56 *$ & 0.16 & 0.57 \\
\hline 5 CISA HTEM $10212 \times$ HD 2967 & 0.15 & 0.71 & -0.97 & -0.88 & $1.557 * *$ & $-8.11 * *$ & $-2.32 * *$ & 0.08 & -0.18 & -0.97 & -2.84 \\
\hline WH 1022 x RAJ 1972 & $-1.99 * *$ & $-3.63 * *$ & $-1.88 * *$ & $5.03 * *$ & $3.44 * *$ & -0.77 & 0.15 & -0.28 & $-0.87 * *$ & 0.49 & 1.46 \\
\hline WH 1022 x PBW 343 & 1.21 & 1.21 & 0.92 & $2.96 * *$ & $5.77 * *$ & $5.47 * *$ & 0.58 & -0.06 & $0.45 *$ & $2.18 * *$ & $6.53 * *$ \\
\hline WH 1022 x 5 CISA HTEM 10211 & $-1.68 *$ & $-1.59 *$ & $-2.95 * *$ & $5.77 * *$ & $6.82 * *$ & $-9.17 * *$ & 0.30 & $-1.35 * *$ & $0.68 * *$ & -0.39 & -1.18 \\
\hline WH 1022 x 46 IBWSN 1005 & $-3.81 * *$ & $-4.29 * *$ & $-2.46 * *$ & -0.73 & $-3.54 * *$ & $-3.17 * *$ & $-3.11 * *$ & $0.99 * *$ & $0.89 * *$ & 0.59 & 1.75 \\
\hline WH 1022 x DBW 14 & $2.39 * *$ & 0.87 & $1.46 *$ & $3.01 * *$ & $3.94 * *$ & $-3.29 * *$ & -0.25 & 0.35 & $0.92 * *$ & -0.84 & -2.53 \\
\hline 5 CISA HTEM 10212 x GW 322 & $1.95 * *$ & 0.63 & 0.57 & 0.55 & $1.49 * *$ & $-16.43 * *$ & -1.16 & $0.90 * *$ & $0.70 * *$ & $-1.8 * *$ & $-5.33 * *$ \\
\hline 5 CISA HTEM 10212 x 3 SATYN 9402 & $1.73 *$ & $2.15 * *$ & $2.64 * *$ & $3.43 * *$ & $6.63 * *$ & $-11.67 * *$ & 1.44 & $-2.98 * *$ & $-1.91 * *$ & $-3.34 * *$ & $-9.95 * *$ \\
\hline 5 CISA HTEM 10212 x K9107 & 0.03 & -0.05 & -0.53 & $4.58 * *$ & $3.56 * *$ & $-8.43 * *$ & -0.02 & -0.14 & $0.56 *$ & 0.16 & 0.57 \\
\hline 5 CISA HTEM 10212 x HD 2967 & 0.15 & 0.71 & -0.97 & -0.88 & $1.557 * *$ & $-8.11 * *$ & $-2.32 * *$ & 0.08 & -0.18 & -0.97 & -2.84 \\
\hline 5 CISA HTEM $10212 \times$ RAJ 1972 & $-3.87 * *$ & $-2.91 * *$ & $-2.27 * *$ & 0.63 & $-2.40 * *$ & $2.20 * *$ & $1.96 *$ & $2.10 * *$ & -0.39 & $2.04 * *$ & $6.20 * *$ \\
\hline 5 CISA HTEM 10212 x PBW 343 & 0.63 & 0.13 & $1.57 *$ & $-2.45 * *$ & $-3.40 * *$ & 0.40 & $-1.94 *$ & $-1.19 * *$ & $-1.34 * *$ & -0.94 & -2.73 \\
\hline $\begin{array}{l}5 \text { CISA HTEM } 10212 \text { x } 5 \text { CISA HTEM } \\
10211\end{array}$ & 1.34 & 0.45 & 0.43 & $2.36 * *$ & -0.68 & $12.96 * *$ & -0.56 & 0.43 & $1.15 * *$ & $-2.18 * *$ & $-6.44 * *$ \\
\hline
\end{tabular}




\begin{tabular}{|c|c|c|c|c|c|c|c|c|c|c|c|}
\hline Cross & $\begin{array}{l}\text { Chlorophyll } \\
\text { content at } \\
\text { booting stage }\end{array}$ & $\begin{array}{l}\text { Chlorophyll } \\
\text { content at } \\
\text { anthesis stage }\end{array}$ & $\begin{array}{l}\text { Chlorophyll } \\
\text { content at grain } \\
\text { filling stage }\end{array}$ & $\begin{array}{l}\text { Days to } \\
\text { heading }\end{array}$ & $\begin{array}{l}\text { Days to } \\
\text { maturity }\end{array}$ & $\begin{array}{l}\text { Plant } \\
\text { height }\end{array}$ & $\begin{array}{l}\text { Number of } \\
\text { productive tiller } \\
\text { per meter }\end{array}$ & $\begin{array}{l}\text { Spike } \\
\text { length }\end{array}$ & $\begin{array}{l}\text { Awn } \\
\text { length }\end{array}$ & $\begin{array}{l}\text { Number of } \\
\text { spikelet's } \\
\text { per spike }\end{array}$ & $\begin{array}{l}\text { Number of } \\
\text { grains per } \\
\text { spike }\end{array}$ \\
\hline 5 CISA HTEM 10212 x 46 IBWSN 1005 & 1.07 & 0.40 & 1.32 & -0.80 & $-2.71 * *$ & $6.64 * *$ & 0.70 & -0.17 & 0.20 & $1.47 * *$ & $4.48 * *$ \\
\hline 5 CISA HTEM 10212 x DBW 14 & -0.62 & -0.47 & $1.47 *$ & $1.27 * *$ & $1.44 *$ & $6.97 * *$ & 1.56 & $-1.01 * *$ & $-0.76 * *$ & 0.71 & 2.21 \\
\hline GW 322 x 3 SATYN 9402 & $-3.05 * *$ & $-3.54 * *$ & $-2.69 * *$ & $-2.00 * *$ & $6.80 * *$ & $2.28 * *$ & $-1.85 *$ & $-0.78 * *$ & -0.39 & $-1.95 * *$ & $-5.86^{* *}$ \\
\hline GW 322 x 2 K9107 & $2.51 * *$ & $1.90 * *$ & $2.74 * *$ & $-2.52 * *$ & $-1.94 * *$ & $3.38 * *$ & -1.30 & $-1.28 * *$ & -0.29 & -0.44 & -1.34 \\
\hline GW 322 x HD 2967 & $-7.19 * *$ & $-9.01 * *$ & $-7.30 * *$ & 0.03 & $-2.94 * *$ & $-6.43 * *$ & -0.94 & 0.04 & $-0.66 * *$ & $-3.58 * *$ & $-10.75 * *$ \\
\hline GW 322 x RAJ 1972 & $1.75 *$ & $1.46 *$ & $1.40 *$ & $1.20 *$ & $9.77 * *$ & -0.59 & 0.68 & -0.27 & $-0.44 *$ & $1.44 * *$ & $4.29 * *$ \\
\hline GW 322 x PBW 343 & -1.18 & -1.14 & -1.16 & $3.79 * *$ & $10.77 * *$ & 0.68 & -0.23 & $1.24 * *$ & $0.55 *$ & $2.46 * *$ & $7.36 * *$ \\
\hline GW 322 x 5 CISA HTEM 10211 & $3.83 * *$ & $3.95 * *$ & $3.57 * *$ & $3.60 * *$ & 0.49 & $-4.20 * *$ & 0.15 & -0.07 & -0.17 & 0.55 & 1.65 \\
\hline GW 322 x 46 IBWSN 1005 & $2.19 * *$ & $1.82 *$ & $1.72 *$ & $2.43 * *$ & -0.21 & -0.89 & 0.41 & -0.21 & $0.42 *$ & 0.20 & 0.58 \\
\hline GW 322 x DBW 14 & 0.87 & 1.20 & $2.07 * *$ & $1.17 *$ & $-3.73 * *$ & -0.35 & 0.27 & $0.83 * *$ & 0.12 & $3.11 * *$ & $9.30 * *$ \\
\hline 3 SATYN 9402 x K9107 & 1.39 & $1.63 *$ & 0.95 & $-4.64 * *$ & $-2.80 * *$ & 1.47 & $2.63 * *$ & $2.65 * *$ & $2.06 * *$ & $1.62 * *$ & $4.84 * *$ \\
\hline 3 SATYN 9402 x HD 2967 & $3.41 * *$ & $3.03 * *$ & $2.91 * *$ & $2.24 * *$ & 0.53 & $3.62 * *$ & $2.65 * *$ & $1.27 * *$ & $1.23 * *$ & $1.05 *$ & $3.13 *$ \\
\hline 3 SATYN 9402 x RAJ 1972 & $1.69 *$ & $1.91 * *$ & 1.11 & $1.41 * *$ & $-10.75 * *$ & $8.43 * *$ & $2.27 * *$ & 0.56 & $0.71 * *$ & -0.77 & -2.33 \\
\hline 3 SATYN 9402 x PBW 343 & -0.34 & 0.26 & 0.24 & $2.01 * *$ & 0.247 & $-2.00 *$ & -0.97 & 0.34 & $-0.54 *$ & -0.42 & -1.26 \\
\hline 3 SATYN 9402 x 5 CISA HTEM 10211 & $-4.30 * *$ & $-3.77 * *$ & $-2.56 * *$ & $1.82 * *$ & $3.30 * *$ & $-1.94 *$ & 0.75 & 0.52 & 0.12 & 1.01 & 3.03 \\
\hline 3 SATYN 9402 x 46 IBWSN 1005 & $-1.74 *$ & $-2.15 * *$ & $-3.57 * *$ & $1.65 * * *$ & $4.94 * *$ & $-4.81 * *$ & $-3.66 * *$ & $1.79 * *$ & 0.28 & 0.99 & 2.95 \\
\hline 3 SATYN 9402 x DBW 14 & 1.08 & 0.94 & -0.45 & 0.39 & -0.92 & $2.64 * *$ & 0.53 & $-1.31 * *$ & $-0.83 * *$ & $1.17 *$ & $3.48 *$ \\
\hline K9107 x HD 2967 & $2.51 * *$ & $2.36^{*}$ & $2.70 * *$ & $1.39 * *$ & $2.46 * *$ & $-3.54 * *$ & -1.13 & $-1.26 * *$ & -0.07 & $-1.61 * *$ & $-4.85 * *$ \\
\hline K9107 x RAJ 1972 & $-2.11 * *$ & $-3.33 * *$ & $-2.03 * *$ & 0.89 & -0.16 & $10.04 * *$ & 0.82 & $0.99 * *$ & $0.48 *$ & $2.07 * *$ & $6.19 * *$ \\
\hline K9107 x PBW 343 & $-2.78 * *$ & $-5.79 * *$ & $-4.40 * *$ & $4.15 * *$ & $4.84 * *$ & $-10.19 * *$ & $1.91 *$ & $-2.06 * *$ & $-1.43 * *$ & 0.43 & 1.26 \\
\hline K9107 x 5 CISA HTEM 10211 & $-1.70 *$ & 0.23 & 0.36 & $0.96 *$ & 0.89 & $5.67 * *$ & $-1.71 *$ & 0.256 & 0.12 & 0.52 & 1.55 \\
\hline K910746 x IBWSN 1005 & $-8.11 * *$ & $-8.11 * *$ & $-6.98 * *$ & $1.79 * *$ & $-2.13 * *$ & $4.67 * *$ & $-2.78 * *$ & $-1.944 * *$ & $-0.82 * *$ & -0.50 & -1.53 \\
\hline K9107 x DBW 14 & $-3.16 * *$ & $-2.71 * *$ & $-3.69 * *$ & -0.14 & $2.68 * *$ & $-3.22 * *$ & $-2.25 * *$ & -0.08 & 0.41 & -0.26 & -0.80 \\
\hline HD $2967 \times$ RAJ 1972 & $3.52 * *$ & $3.54 * *$ & $2.86 * *$ & $1.77 * *$ & -0.82 & $-3.54 * *$ & $-2.49 * *$ & $-0.89 * *$ & $-1.19 * *$ & $-1.07 *$ & $-3.22 *$ \\
\hline HD $2967 \times$ PBW 343 & $-2.52 * *$ & $-2.19 * *$ & $-2.90 * *$ & $-2.64 * *$ & $-2.16 * *$ & $4.95 * *$ & -0.40 & 0.49 & 0.07 & -0.71 & -2.15 \\
\hline HD 2967 x 5 CISA HTEM 10211 & $-3.53 * *$ & $-3.48 * *$ & $-3.01 * *$ & $-2.50 * *$ & 0.56 & $3.22 * *$ & -1.35 & 0.21 & 0.39 & $1.39 * *$ & $4.14 * *$ \\
\hline HD 2967 x 46 IBWSN 1005 & $2.62 * *$ & $2.05 * *$ & 1.18 & $-1.66 * *$ & -0.80 & $7.66 * *$ & $3.25 * *$ & $0.81 * *$ & $0.58 * *$ & $1.69 * *$ & $5.07 * *$ \\
\hline HD $2967 \times$ DBW 14 & $-4.13 * *$ & $-4.07 * *$ & $-2.73 * *$ & $-4.26 * *$ & $1.34 *$ & $4.13 * *$ & $4.44 * *$ & $0.97 * *$ & $-0.69 * *$ & $1.60 * *$ & $4.80 * *$ \\
\hline RAJ 1972 x PBW 343 & 1.43 & $1.82 *$ & 1.33 & $2.86 * *$ & $4.22 * *$ & 0.60 & -1.11 & 0.41 & $0.45 *$ & 0.97 & 2.89 \\
\hline RAJ 1972 x 5 CISA HTEM 10211 & $-4.02 * *$ & $-3.02 * *$ & $-3.87 * *$ & -0.33 & $5.94 * *$ & $-9.01 * *$ & $-2.06 *$ & -0.07 & 0.20 & -0.94 & -2.83 \\
\hline PBW 343 x 46 IBWSN 1005 & 0.03 & 1.26 & 0.82 & $-2.57 * *$ & $-4.09 * *$ & -0.46 & -1.37 & $1.64 * *$ & $1.21 * *$ & $-1.27 *$ & $-3.83 *$ \\
\hline PBW 343 x DBW 14 & $2.92 * *$ & $3.54 * *$ & $3.10 * *$ & $-2.16 * *$ & $5.72 * *$ & $2.91 * *$ & -1.52 & -0.29 & $-0.45 *$ & $-1.36 *$ & $-4.10 * *$ \\
\hline 5 CISA HTEM 10211 x 46 IBWSN 1005 & $2.94 * *$ & $1.98 * *$ & $1.58 *$ & -0.42 & $1.96 * *$ & $-5.80 * *$ & 1.01 & $-1.64 * *$ & $-1.40 * *$ & -0.84 & -2.54 \\
\hline 5 CISA HTEM 10211 x DBW 14 & 0.90 & -0.08 & -0.40 & $3.32 * *$ & $3.77 * *$ & $10.27 * *$ & -0.13 & $-0.64 *$ & -0.13 & 0.40 & 1.19 \\
\hline 46 IBWSN 1005 x DBW 14 & -0.65 & 0.56 & 0.19 & -0.85 & $4.75 * *$ & $-1.69 *$ & $1.80 *$ & -0.01 & 0.33 & -0.62 & -1.88 \\
\hline Sij-Sik 95\% & 2.14 & 2.04 & 1.20 & 1.35 & 1.64 & 2.22 & 2.31 & 0.85 & 0.62 & 1.52 & 4.53 \\
\hline Sij-Sik 99\% & 2.82 & 2.70 & 2.64 & 1.79 & 2.17 & 2.93 & 3.05 & 1.12 & 0.82 & 2.01 & 5.98 \\
\hline Sij-Skm 95\% & 2.05 & 1.96 & 1.92 & 1.30 & 1.58 & 2.14 & 2.22 & 0.82 & 0.60 & 1.46 & 4.35 \\
\hline Sij-Skm 99\% & 2.71 & 2.59 & 2.53 & 1.72 & 2.08 & 2.82 & 2.93 & 1.08 & 0.79 & 1.93 & 5.74 \\
\hline
\end{tabular}




\begin{tabular}{|c|c|c|c|c|c|c|c|c|c|c|}
\hline Cross & $\begin{array}{l}\text { Thousand } \\
\text { grain weight }\end{array}$ & $\begin{array}{l}\text { Grain } \\
\text { yield }\end{array}$ & Biomass & $\begin{array}{l}\text { Harvest } \\
\text { index }\end{array}$ & $\begin{array}{l}\text { Grain } \\
\text { protein } \\
\text { content }\end{array}$ & $\begin{array}{l}\text { Nitrogen } \\
\text { harvest } \\
\text { index }\end{array}$ & $\begin{array}{l}\text { Total } \\
\text { nitrogen } \\
\text { uptake }\end{array}$ & $\begin{array}{l}\text { Nitrogen } \\
\text { uptake } \\
\text { efficiency }\end{array}$ & $\begin{array}{l}\text { Nitrogen } \\
\text { utilization } \\
\text { efficiency }\end{array}$ & $\begin{array}{l}\text { Nitrogen } \\
\text { use } \\
\text { efficiency }\end{array}$ \\
\hline 2 WYCYT 34 x WH 1022 & 2.21 & 0.55 & -1.42 & $2.45 *$ & 0.22 & -1.45 & 0.02 & 0.02 & -0.09 & 0.70 \\
\hline 2 WYCYT 34 x 5 CISA HTEM 10212 & $3.26 *$ & 0.99 & 1.32 & 1.30 & -0.03 & 0.51 & 0.02 & 0.02 & 1.03 & 1.27 \\
\hline 2 WYCYT 34 x GW 322 & -0.93 & 0.80 & -0.63 & $2.55 *$ & 0.32 & 1.08 & 0.01 & 0.01 & 1.36 & 1.02 \\
\hline 2 WYCYT 34 x 3 SATYN 9402 & $-6.24 * *$ & $-1.38 *$ & $3.22 *$ & $-5.75 * *$ & -0.52 & $-2.48 *$ & 0.01 & 0.01 & $-3.07 * *$ & $-1.77 *$ \\
\hline 2 WYCYT 34 x K9107 & -0.24 & $-1.88 * *$ & -0.82 & $-4.11 * *$ & -0.23 & -0.47 & -0.03 & -0.04 & -1.94 & $-2.40 * *$ \\
\hline 2 WYCYT 34 x HD 2967 & -1.67 & -0.22 & 2.16 & $-2.78 *$ & 0.05 & -0.72 & 0.02 & 0.03 & -1.87 & -0.28 \\
\hline 2 WYCYT 34 x RAJ 1972 & -2.68 & 0.51 & -2.10 & $2.95 *$ & -0.42 & -0.63 & -0.02 & -0.02 & $2.19 *$ & 0.65 \\
\hline 2 WYCYT 34 x PBW 343 & $-3.83 *$ & 0.19 & 0.33 & 0.22 & -0.14 & 0.22 & -0.01 & -0.001 & 0.55 & 0.25 \\
\hline 2 WYCYT 34 x 5 CISA HTEM 10211 & 0.48 & 0.41 & $-3.09 *$ & $3.28 * *$ & -0.22 & 0.09 & -0.03 & -0.04 & $2.60 *$ & 0.52 \\
\hline 2 WYCYT 34 x 46 IBWSN 1005 & $3.42 *$ & -0.39 & $5.18 * *$ & $-5.08 * *$ & 0.26 & 0.24 & $0.04 *$ & $0.05 *$ & $-3.35 * *$ & -0.51 \\
\hline 2 WYCYT 34 x DBW 14 & 1.29 & 0.54 & $4.58 * *$ & -1.43 & -0.47 & 0.55 & 0.02 & 0.03 & -0.09 & 0.70 \\
\hline WH 1022 x 5 CISA HTEM 10212 & -3.01 & $1.20 *$ & 2.76 & 0.80 & 0.38 & 1.43 & 0.03 & 0.05 & 0.50 & $1.53 *$ \\
\hline WH 1022 x GW 322 & 0.77 & $1.17 *$ & 2.04 & 1.37 & 0.41 & 0.12 & $0.04 *$ & $0.05 *$ & -0.21 & $1.50 *$ \\
\hline WH 1022 x 3 SATYN 9402 & -1.86 & -0.62 & -0.45 & -1.10 & -0.24 & $-2.67 * *$ & 0.00 & 0.003 & -1.38 & -0.80 \\
\hline WH 1022 x K9107 & 0.87 & $1.97 * *$ & 0.93 & $4.18 * *$ & -0.52 & -0.25 & 0.02 & 0.02 & $3.47 * *$ & $2.52 * *$ \\
\hline WH 1022 x HD 2967 & -1.29 & $-1.31 *$ & $4.82 * *$ & $-7.32 * *$ & -0.10 & -1.14 & 0.02 & 0.03 & $-4.60 * *$ & $-1.67 *$ \\
\hline WH 1022 x RAJ 1972 & -1.16 & -0.45 & -1.50 & 0.06 & -0.20 & 0.54 & -0.03 & -0.04 & 0.93 & -0.58 \\
\hline WH 1022 x PBW 343 & -1.18 & $-1.11 *$ & -0.24 & $-2.62 *$ & -0.21 & 1.54 & -0.04 & -0.04 & -0.16 & $-1.42 *$ \\
\hline WH 1022 x 5 CISA HTEM 10211 & -0.02 & 0.63 & $-4.93 * *$ & $6.19 * *$ & 0.23 & $2.06 *$ & -0.04 & -0.04 & $4.13 * *$ & 0.80 \\
\hline WH 1022 x 46 IBWSN 1005 & $-3.77 *$ & -0.98 & -0.40 & $-2.68 *$ & 0.34 & 1.89 & -0.02 & -0.03 & -0.98 & -1.26 \\
\hline WH 1022 x DBW 14 & 1.48 & $-1.88 * *$ & $-2.78 *$ & $-2.62 *$ & -0.62 & -0.33 & $-0.05 *$ & $-0.07 *$ & -0.80 & $-2.41 * *$ \\
\hline 5 CISA HTEM 10212 x GW 322 & 0.003 & 0.40 & 2.46 & -1.02 & 0.08 & -0.69 & 0.03 & 0.04 & -1.47 & 0.51 \\
\hline 5 CISA HTEM 10212 x 3 SATYN 9402 & -2.82 & $-2.46 * *$ & $-4.26 * *$ & $-2.45 *$ & -0.28 & 0.29 & $-0.08 * *$ & $-0.1 * *$ & -0.04 & $-3.16 * *$ \\
\hline 5 CISA HTEM 10212 x K9107 & 0.56 & -0.71 & -0.03 & -1.80 & -0.07 & -1.25 & 0.0001 & 0.001 & -1.60 & -0.91 \\
\hline 5 CISA HTEM 10212 x HD 2967 & -1.56 & -0.63 & -0.56 & -1.53 & -0.56 & -0.94 & -0.02 & -0.03 & 0.13 & -0.80 \\
\hline 5 CISA HTEM 10212 x RAJ 1972 & $3.97 *$ & 0.36 & $-3.61 *$ & $3.99 * *$ & -0.20 & -0.65 & -0.02 & -0.03 & $2.23 *$ & 0.47 \\
\hline 5 CISA HTEM $10212 \times$ PBW 343 & 1.75 & 0.32 & -1.71 & $2.39 *$ & -0.06 & -0.700 & -0.002 & -0.01 & 0.86 & 0.41 \\
\hline 5 CISA HTEM 10212 x 5 CISA HTEM 10211 & 1.40 & 0.28 & -0.47 & 1.12 & 0.46 & 0.01 & 0.02 & 0.02 & -0.42 & 0.37 \\
\hline 5 CISA HTEM 10212 x 46 IBWSN 1005 & 2.19 & -0.48 & $4.63 * *$ & $-5.43 * *$ & 0.13 & 0.99 & 0.02 & 0.03 & $-2.73 *$ & -0.62 \\
\hline 5 CISA HTEM 10212 x DBW 14 & 1.22 & 0.46 & $-3.014 *$ & $4.37 * *$ & -0.58 & -1.61 & -0.02 & -0.02 & $2.60 *$ & 0.58 \\
\hline GW 322 x 3 SATYN 9402 & -1.80 & $-2.46 * *$ & -2.19 & $-4.44 * *$ & -0.37 & -0.50 & $-0.06 * *$ & $-0.08 * *$ & -1.38 & $-3.15 * *$ \\
\hline GW 322 x K9107 & 0.85 & $-2.03 * *$ & -2.38 & $-3.34 * *$ & -0.11 & -0.86 & $-0.04 *$ & $-0.05 *$ & -1.96 & $-2.60 * *$ \\
\hline GW 322 x HD 2967 & -0.40 & -0.69 & 2.05 & $-3.89 * *$ & -0.08 & 0.54 & 0.00 & 0.00 & -1.39 & -0.88 \\
\hline GW 322 x RAJ 1972 & 2.95 & -0.42 & 0.66 & -1.60 & 0.11 & 0.36 & 0.00 & 0.00 & -1.19 & -0.54 \\
\hline GW 322 x PBW 343 & 2.78 & 0.14 & -0.02 & 0.36 & -0.61 & -0.38 & -0.01 & -0.02 & 1.37 & 0.18 \\
\hline GW 322 x 5 CISA HTEM 10211 & $6.06 * *$ & 0.52 & 0.83 & 0.59 & 0.00 & -0.10 & 0.01 & 0.02 & 0.26 & 0.67 \\
\hline GW 322 x 46 IBWSN 1005 & $-3.72 *$ & -0.56 & $-2.89 *$ & 0.99 & 0.11 & 1.07 & -0.03 & -0.04 & 1.19 & -0.71 \\
\hline GW 322 x DBW 14 & 2.99 & 1.02 & 2.75 & 0.58 & -0.17 & 0.29 & 0.03 & 0.04 & 0.34 & 1.31 \\
\hline
\end{tabular}




\begin{tabular}{|c|c|c|c|c|c|c|c|c|c|c|}
\hline 3 SATYN 9402 x K9107 & 1.12 & $2.17 * *$ & 2.30 & $2.94 *$ & 0.16 & 0.25 & $0.05 * *$ & $0.06 *$ & 0.82 & $2.78 * *$ \\
\hline Cross & $\begin{array}{l}\text { Thousand } \\
\text { grain weight }\end{array}$ & $\begin{array}{l}\text { Grain } \\
\text { yield }\end{array}$ & Biomass & $\begin{array}{l}\text { Harvest } \\
\text { index }\end{array}$ & $\begin{array}{l}\text { Grain } \\
\text { protein } \\
\text { content }\end{array}$ & $\begin{array}{l}\text { Nitrogen } \\
\text { harvest } \\
\text { index }\end{array}$ & $\begin{array}{l}\text { Total } \\
\text { nitrogen } \\
\text { uptake }\end{array}$ & $\begin{array}{l}\text { Nitrogen } \\
\text { uptake } \\
\text { efficiency }\end{array}$ & $\begin{array}{l}\text { Nitrogen } \\
\text { utilization } \\
\text { efficiency }\end{array}$ & $\begin{array}{l}\text { Nitrogen } \\
\text { use } \\
\text { efficiency }\end{array}$ \\
\hline 3 SATYN 9402 x HD 2967 & 1.11 & $2.26 * *$ & $3.00 *$ & $2.43 *$ & 0.18 & 0.49 & $0.06 * *$ & $0.07 * *$ & 0.87 & $2.89 * *$ \\
\hline 3 SATYN 9402 x RAJ 1972 & $5.6 * *$ & -0.36 & 0.71 & -1.49 & -0.08 & 0.35 & -0.01 & -0.01 & -0.35 & -0.46 \\
\hline 3 SATYN 9402 x PBW 343 & -2.91 & 0.88 & $3.56 *$ & -1.00 & 0.05 & 0.80 & 0.03 & 0.04 & 0.09 & 1.12 \\
\hline 3 SATYN $x 5$ CISA HTEM 10211 & 1.52 & $1.20 *$ & 0.11 & $2.83 *$ & 0.54 & 1.69 & 0.02 & 0.03 & 1.35 & $1.540 *$ \\
\hline 3 SATYN 9402 x 46 IBWSN 1005 & $5.19 * *$ & $1.60 * *$ & 0.67 & $3.11 * *$ & 0.41 & 1.65 & 0.03 & 0.04 & 1.26 & $2.052 * *$ \\
\hline 3 SATYN 9402 x DBW 14 & $4.18 * *$ & $4.28 * *$ & $3.63 *$ & $7.28 * *$ & 0.54 & $2.06 *$ & $0.09 * *$ & $0.12 * *$ & $3.06 * *$ & $5.49 * *$ \\
\hline K9107 x HD 2967 & 1.90 & -0.45 & -0.39 & -0.88 & -0.19 & -0.03 & -0.02 & -0.02 & 0.04 & -0.57 \\
\hline K9107 x RAJ 1972 & 1.28 & 0.40 & 2.32 & -0.96 & 0.32 & 0.86 & 0.02 & 0.03 & -0.49 & 0.52 \\
\hline K9107 x PBW 343 & -0.56 & 0.29 & -0.26 & 1.18 & 0.39 & 1.49 & 0.001 & 0.00 & 0.57 & 0.37 \\
\hline K9107 x 5 CISA HTEM 10211 & -1.64 & 0.05 & -0.28 & 0.27 & -0.19 & -0.12 & -0.01 & -0.01 & 0.75 & 0.06 \\
\hline K910746 x IBWSN 1005 & -2.48 & -0.09 & 0.85 & -1.20 & 0.02 & 0.46 & -0.001 & 0.002 & -0.30 & -0.11 \\
\hline K9107 x DBW 14 & $-4.34 * *$ & $1.25 *$ & -0.21 & $3.89 * *$ & -0.39 & -0.08 & 0.004 & 0.009 & $2.85 * *$ & $1.60 *$ \\
\hline HD 2967 x RAJ 1972 & -0.60 & -0.003 & -1.52 & 1.01 & 0.20 & 0.63 & -0.01 & -0.01 & 0.68 & -0.004 \\
\hline HD $2967 \times$ PBW 343 & 0.56 & 0.02 & -1.13 & 1.00 & -0.13 & 0.52 & -0.02 & -0.02 & 1.31 & 0.03 \\
\hline HD 2967 x 46 IBWSN 1005 & 2.80 & $2.35 * *$ & 1.25 & $4.67 * *$ & 0.40 & 0.40 & $0.050 *$ & $0.06 *$ & 1.41 & $3.00 * *$ \\
\hline HD $2967 \times$ DBW 14 & 0.80 & $1.52 * *$ & $3.94 * *$ & 0.34 & 0.28 & 0.80 & $0.06 * *$ & $0.07 * *$ & -0.11 & $1.94 * *$ \\
\hline RAJ 1972 x PBW 343 & -2.07 & 0.42 & -0.20 & 1.28 & 0.05 & -0.25 & 0.01 & 0.01 & 0.54 & 0.53 \\
\hline RAJ 1972 x 5 CISA HTEM 10211 & $-4.74 * *$ & 0.12 & $5.29 * *$ & $-3.45 * *$ & -0.15 & -0.52 & 0.04 & 0.04 & -2.03 & 0.15 \\
\hline RAJ 1972 x 46 IBWSN 1005 & -0.72 & -0.17 & 0.29 & -1.19 & 0.08 & -0.43 & 0.01 & 0.01 & -0.83 & -0.22 \\
\hline RAJ 1972 x DBW 14 & 0.60 & 0.30 & -0.75 & 1.55 & 0.04 & 0.04 & 0.01 & 0.01 & 0.49 & 0.39 \\
\hline PBW 343 x 5 CISA HTEM 10211 & 2.25 & -0.23 & -1.86 & 0.91 & -0.17 & -0.76 & -0.01 & -0.02 & 0.46 & -0.30 \\
\hline PBW 343 x 46 IBWSN 1005 & 1.91 & -0.87 & -0.92 & -1.90 & -0.27 & -1.43 & -0.02 & -0.02 & -1.18 & -1.11 \\
\hline PBW $343 \times$ DBW 14 & 1.28 & 0.93 & $2.89 *$ & 0.34 & -0.33 & -0.66 & 0.03 & 0.04 & 0.13 & 1.20 \\
\hline 5 CISA HTEM 10211 x 46 IBWSN 1005 & -1.57 & $-1.21 *$ & 2.66 & $-5.63 * *$ & -0.03 & -0.91 & 0.01 & 0.02 & $-3.39 * *$ & $-1.55 *$ \\
\hline 5 CISA HTEM 10211 x DBW 14 & -1.93 & -0.32 & $4.95 * *$ & $-4.19 * *$ & 0.01 & 0.43 & 0.04 & 0.05 & $-2.83 * *$ & -0.41 \\
\hline 46 IBWSN 1005 x DBW 14 & -0.82 & 0.39 & -1.72 & $2.67 *$ & $-1.11 * *$ & -1.15 & -0.03 & -0.04 & $3.72 * *$ & 0.50 \\
\hline Sij-Sik $95 \%$ & 4.46 & 1.60 & 4.05 & 3.36 & 1.22 & 2.85 & 0.06 & 0.07 & 3.11 & 2.05 \\
\hline Sij-Sik $99 \%$ & 5.89 & 2.11 & 5.34 & 4.44 & 1.61 & 3.76 & 0.08 & 0.10 & 4.10 & 2.71 \\
\hline Sij-Skm 95\% & 4.28 & 1.54 & 3.89 & 3.23 & 1.18 & 2.74 & 0.05 & 0.07 & 2.99 & 1.97 \\
\hline Sij-Skm 99\% & 5.66 & 2.03 & 5.13 & 4.27 & 1.55 & 3.62 & 0.07 & 0.09 & 3.94 & 2.60 \\
\hline
\end{tabular}




\section{Specific combining ability effects of the 66} single crosses

Estimate of sca effects of the sixty-six diallele crosses for morpho-physiological, yield, yield attributes, NUE and its related traits are shown in Table 3. Among sixty-six single crosses, the six cross combinations namely, 3 SATYN $9402 \times$ K9107, 3 SATYN $9402 \times$ HD 2967, 3 SATYN 9402 x 46 IBWSN 1005, 3 SATYN $9402 \times$ DBW 14, HD $2967 \times 46$ IBWSN 1005 and WH 1022 x K 9107 found to be the best specific combiners for yield and NUE. These cross combinations also exhibited high perse performance. Previous researchers, Khan et al., (2010), Farshadfar et al., (2013), and Ahmad et al., (2014) also found specific cross-combinations for different NUE component characteristics. Crosses with high mean and $s c a$ effects are more likely to exhibit transgressive segregation in self-pollinated crops such as wheat, where pure line breeding is cardinal (Ahmad et al., 2014 and Singh et al., 2003). Singh and Chatrath (1992) indicated that if the desirable characteristics are controlled by non-additive gene effects in autogamous crops, the selection should be delayed to subsequent generations. This findings in the inference that transgressive segregants which are potential can be acquired from subsequent generations as proposed by various researchers including Nour et al., (2011), Khaled and Sayem (2014) and Kose et al., (2017).

The NUE parents namely, 3 SATYN 9402, K9107, HD 2967 and 46 IBWSN 1005 found to best general combiner among twelve parents for important traits like grain yield, harvest index, nitrogen utilization efficiency and nitrogen use efficiency. The crosses namely 3 SATYN 9402 x K9107, 3 SATYN 9402 x HD 2967, 3 SATYN 9402 x 46 IBWSN 1005, 3 SATYN 9402 x DBW 14, HD 2967 x 46 IBWSN 1005 and WH 1022 x
K 9107 found to be the best specific combiners for yield and Nitrogen use efficiency. Therefore Parents with good general combining ability (GCA) may be employed in recombination breeding while $\mathrm{F}_{1} \mathrm{~S}$ with specific combining ability (SCA) effects may be further advanced in selecting high NUE segregants with care in subsequent segregating generations under low-N conditions.

\section{References}

Ahmad, S., Ahtisham, S., Kashif, M. and Ali, Q., 2014, Role of combining ability to develop higher yielding wheat (Triticum aestivum L.) genotypes. Nat. Sci., 12 (11): 155-161.

Ashadusjaman, M., Shamsuddoha, M., Alma, M. J. and Begum, M. O., 2012, Combining ability and gene action for different root characters in spring wheat. J. Environ. Sci. Natural Resources, 5(2): 73-76.

Barraclough, P. B., Howarth, J. R., Jones, J., Bellido, R. L., Parmar, S., Shepherd, C. E. and Hawkesford, M, J., 2010, Nitrogen efficiency of wheat: genotypic and environmental variation and prospects for improvement. Eur. J. Agron., 33: 1-11.

Crews, T. E. and Peoples, M. B., 2004, Legume versus fertilizer sources of nitrogen: ecological tradeoffs and human needs. Agric. Ecosyst. Environ., 102: 279-297.

Farshadfar, E., Rafiee, F. and Hasheminasab, H., 2013, Evaluation of genetic parameters of agronomic and morpho-physiological indicators of drought tolerance in bread wheat (Triticum aestivum L.) using diallel mating design. Aus. J. Crop Sci., 7 (2): 268275.

Foulkes, M. J., Hawkesford, M. J., Barraclough, P. B., Holdsworth, M. J., Kerr, S., Kightley, and Shewry, P. R., 2009. Identifying traits to improve the nitrogen economy of wheat: recent advances and future prospects. Field Crops Res., 114: 329-342.

Gallais, A. and Coque, M., 2005, Genetic variation and selection for nitrogen use efficiency in maize: a synthesis. Maydica, 50: 531-547.

Gorny, A. G., Banasza, K. Z., Lugowska, B. and 
Ratajcka, D., 2011, Inheritance of the efficiency of nitrogen uptake and utilisation in winter wheat (Triticum aestivum L.) under diverse nutritional levels. Euphytica, 177: 191-206.

Griffing, B., 1956, Concept of general and specific combining ability in relation to diallel crossing system. Aus. J. Biol. Sci., 9: 463-493.

Hawkesford, M. J., 2012. The diversity of nitrogen use efficiency for wheat varieties and the potential for crop improvement. $J$. Cereal Sci., 96 (3): 10-12.

Hirel, B., Le Gouis, J., Ney, B. and Gallais, A., 2007. The challenge of improving nitrogen use efficiency in crop plants: towards a more central role for genetic variability and quantitative genetics within integrated approaches. J. Exp. Bot., 58: 2369-2387.

Hirel, B., Le Gouis, J., Ney, B. and Gallais, A., 2007. The challenge of improving nitrogen use efficiency in crop plants: towards a more central role for genetic variability and quantitative genetics within integrated approaches. J. Exp. Bot., 58: 2369-2387.

Joshi, S. K., Sharma, S. N., Singhania, D. L. and Sain, R. S., 2004, Combining ability in the $F_{1}$ and $F_{2}$ generations of diallel cross in hexaploid wheat (Triticum aestivam L. em. Thell). Heriditas., 141: 115-121.

Khan, A. A., Iqbal, A., Awan, F. S. and Khan, I. A., 2010, Genetic diversity in wheat germplasm collections from Balochistan province of Pakistan. Pakistan J. Bot., 42(1): 89-96.

Khan, M. A., Nadeem, A., Muhammad, A., Aziz, U. R. and Iqbal, M. M., 2007. Combining ability analysis in wheat. Pakistan J. Agric. Sci., 44 (1): 1-5.
Kose, A., 2017, Gene action and combining abitiy in line $\times$ tester population of safflower (Carthamus tinctorius L.). Turkish J. F. Crop., 22(2): 197-203.

Mahantashivayogayya, K., Hanchinal, R. R. and Salimath, P. M., 2004. Combining ability in Dicoccum wheat. Karnataka J. Agric. Sci., 17(3): 451-454.

Moll, R. H, Kamprath E. J, and Jackson W. A, 1982, Analysis and interpretation of factors which contribute to efficiency of nitrogen utilization. Agron. J., 74: 562-564.

Nour, A., Nadya, A. R., El-fateh, H. S. A. and Mostafa, A. K., 2011, Line $\times$ tester analysis for yield and its traits in bread wheat. Egypt. J. Agric. Res., 89(3): 979-992.

Singh, G., Kulshreshtha, N., Singh, B. N., Setter, T. L., Singh, M. K., Saharan, M. S, Tyagi, B. S., Verma Ajay and Sharma, I., 2014, Germplasm characterization, association and clustering for salinity and waterlogging tolerance in bread wheat (Triticum aestivum). Indian J. Agric. Sci., 84: 110210.

Singh, K. N. and Chatrath, R., 1992, Genetic variability in grain yield and its component characters and their associations under salt stress conditions in tissue culture lines of bread wheat (Triticum aestivum L.), Wheat Inform. Ser., 75(2): 46-53.

Singh, S. P., Singh, L. R., Devendra, S. and Rajendra, K., 2003, Combining ability in common wheat (Triticum aestivum L.) grown in sodic soil. Prog Agric., 3 (1/2): 78-80.

Sprague, G. F. and Tatum, L. A., 1942, General versus specific combining ability in single crosses of corn. J. A. S. Agron., 34: 923932.

\section{How to cite this article:}

Mahalaxmi K. Patil and Desai, S.A. 2019. Combining Ability Studies for MorphoPhysiological, Yield, Yield Attributes Nitrogen Use Efficiency and Its Related Traits in Bread Wheat (Triticum aestivum L.). Int.J.Curr.Microbiol.App.Sci. 8(08): 976-986. doi: https://doi.org/10.20546/ijcmas.2019.808.113 